\title{
Qualidade de frutos de acessos de umbu-cajazeira (Spondias sp.)
}

\author{
Perla J. S. Gondim', Silvanda de M. Silva', Walter E. Pereira', \\ Ana L. Dantas', José R. Chaves Neto' \& Luana F. dos Santos ${ }^{1}$
}

\begin{abstract}
RESUMO
Os frutos da umbu-cajazeira (Spondias sp.) são amplamente consumidos frescos ou processados, em decorrência de suas características sensoriais, apresentando-se com boas perspectivas de cultivo e comercialização. No entanto, a avaliação da qualidade pós-colheita de frutos de acessos das suas áreas de ocorrência se faz necessária, no sentido não apenas de identificar materiais com características promissoras de interesse para a indústria ou consumo fresco, mas também para dar suporte aos programas de melhoramento. Neste trabalho foram avaliados frutos colhidos na maturidade comercial (coloração totalmente amarela) de oito acessos provenientes do município de Areia, Brejo paraibano, quanto às características físicas e físico-químicas. A massa fresca média dos frutos dos acessos avaliados foi de 28,82 g e o rendimento médio em polpa foi de $68,5 \%$. Com base na análise de componentes principais, o acesso A2 se destacou por apresentar conteúdos superiores de sólidos solúveis (12,9\%) e açúcares redutores (10,9\%), mais baixa acidez titulável $(0,94 \%$ de ácido cítrico) e superior relação SS/AT $(13,75)$, indicando que este acesso pode ser uma alternativa viável, tanto para o mercado de frutas frescas como para o processamento.
\end{abstract}

Palavras-chave: pós-colheita, características físicas, características físico-químicas, padrões de identidade e qualidade

\section{Fruit quality of 'umbu-caja' (Spondias sp.) access}

\begin{abstract}
Fruits of 'umbu-caja' (Spondias sp.) are widely consumed fresh or processed, due to their appreciated sensorial characteristics, presenting great perspectives for cultivation and marketing. However, postharvest quality evaluation of fruits of access occurring in areas is needed seeking to identify material of interest for the industry or fresh consumption, as well as to provide support for breeding programs. In this study quality of fruits harvested at the commercial maturity of eight access, from municipality of Areia in 'Brejo paraibano' was evaluated, as for the physical and physicochemical characteristics. The mean fresh mass for fruits from all evaluated access was $28.82 \mathrm{~g}$ and the pulp yield was $68.5 \%$. Based on principal component analysis, the A2 access was highlighted by presenting higher contents of soluble solids (12.9\%) and reducing sugars $(10.9 \%)$, lower titratable acidity $(0.94 \%$ of citric acid), and higher SS/TA ratio (13.75), indicating that access can be a reliable alternative for fresh fruit market and processing.
\end{abstract}

Key words: postharvest, physical characteristics, physicochemical characteristics, identity and quality standards 


\section{INTRODUÇÃO}

O Brasil apresenta uma das maiores diversidade de espécies frutíferas do mundo, algumas das quais ainda são desconhecidas e outras pouco exploradas. Portanto, estudos integrados são necessários no sentido de introduzir essas espécies nativas e não tradicionais na cadeia produtiva. Neste sentido e embora o país se destaque como terceiro maior produtor mundial de frutas entre as dez frutíferas mais produzidas, nenhuma é nativa (Souza et al., 2006; Ritzinger et al., 2008).

Portanto, das frutíferas nativas brasileiras a umbucajazeira (Spondias sp.) ainda é uma planta em domesticação, considerada híbrido natural entre o umbuzeiro (Spondias tuberosa Arr. Cam.) e a cajazeira (Spondias mombin L.) (Santana et al., 2011) cujos frutos, denominados umbu-cajá, possuem elevado potencial de cultivo (Silva Júnior et al., 2004). No Nordeste brasileiro a polpa do umbu-cajá apresenta demanda crescente entre as polpas comercializadas (Silva \& Koblitz, 2010); contudo, sua industrialização é dependente da variação sazonal levando-se em conta a exploração extrativista e as práticas deficientes de colheita e pós-colheita. Assim, esta demanda crescente pela polpa do umbu-cajá tem despertado interesse relevante para o cultivo desta espécie, embora pouca informação esteja disponível quanto ao estabelecimento de pomares comerciais (Santana et al., 2011). Sua inclusão como espécie na agricultura moderna exige, entretanto, a identificação de material propagado cujos acessos, além de apresentarem elevada capacidade produtiva (Souza et al., 2006; Ritzinger et al., 2008; Santos et al., 2010), seus frutos também devem diferir quanto aos aspectos de qualidade (Soares et al., 2006; Carvalho et al., 2008; Silva et al., 2011; 2012).

Com base no exposto objetivou-se, neste trabalho, avaliar a qualidade de frutos de acessos da umbu-cajazeira provenientes do brejo paraibano buscando identificar materiais promissores.

\section{Material e MÉTOdos}

O trabalho foi realizado com frutos colhidos manualmente de toda extensão da copa, nas primeiras horas do dia. A colheita foi realizada quando o fruto havia atingido a maturidade comercial, caracterizada pela coloração da casca totalmente amarela, antes da abscisão da planta (Lima et al., 2002), de oito acessos de umbu-cajazeira (Spondias sp.) de ocorrência espontânea no município de Areia, $\mathrm{PB}$, denominados, neste trabalho: A1, A2, A3, A4, A5, A6, A7 e A8. O município de Areia é localizado na Microrregião do Brejo Paraibano, com altitude local de $534,86 \mathrm{~m}$ situando-se entre as coordenadas geográficas $6^{\circ} 58^{\prime} 12$ " de latitude sul e $35^{\circ} 42^{\prime} 15^{\prime \prime}$ de longitude Oeste de Greenwich, com clima quente e úmido. A temperatura da região oscila entre a máxima de $34{ }^{\circ} \mathrm{C}$ e a mínima de $18{ }^{\circ} \mathrm{C}$ com precipitação média anual de $2000 \mathrm{~mm}$.

Após a colheita os frutos foram transportados ao Laboratório de Biologia e Tecnologia Pós-Colheita, no Centro de Ciências Agrárias (CCA), da Universidade Federal da Paraíba (UFPB). Após lavagem e sanitização foram separados 25 frutos (cada fruto considerado uma repetição), de cada acesso, para caracterização física: massa fresca do fruto $(\mathrm{g})$, percentagem da casca e semente. Os frutos foram pesados individualmente em balança semianalítica (MARK 3100). A massa da polpa foi considerada a diferença entre a massa do fruto e a da casca + semente; o rendimento foi considerado o peso da casca + peso da polpa; enfim, o comprimento $(\mathrm{mm})$ e o diâmetro $(\mathrm{mm})$ foram obtidos com auxílio de paquímetro digital (Within $300 \mathrm{~mm}$ ).

Para as avaliações físico-químicas foram utilizadas 3 repetições de cerca de $250 \mathrm{~g}$ de polpa de cada acesso e homogeneizadas para as determinações de: sólidos solúveis (\% SS): determinados com refratômetro digital (Krüss-Optronic, Hamburgo, Alemanha); pH: determinado com potenciômetro digital (Hanna, Singapura) e amido (g.100-1 g polpa): extração feita por hidrólise ácida, conforme a técnica da AOAC (2000); acidez titulável (\% AT): por titulometria com $\mathrm{NaOH} 0,1 \mathrm{M}$, segundo Brasil (2008), expressa em percentagem de ácido cítrico; relação SS/AT: relação entre os SS e AT; açúcares redutores ( $\left(\mathrm{g} 100 \mathrm{~g}^{-1} \mathrm{polpa}\right)$ : realizada segundo Silva et al. (2011) utilizando o ácido 3,5 dinitrosalicílico (DNS).

O delineamento experimental utilizado foi o inteiramente casualizado. Os dados foram submetidos à análise de variância (ANOVA) utilizando-se o software SAS (9.2) sem transformação. A comparação das médias dos acessos foi realizada pelo teste de Scott-Knott a 0,05 de probabilidade. $\mathrm{Na}$ análise multivariada fez-se uso da análise de componentes principais avaliando a resposta aos fatores estudados para o autovalor da matriz de correlação $(\lambda)$.

\section{Resultados e Discussão}

A amplitude para o comprimento e diâmetro de frutos de diferentes acessos de umbu-cajazeira variou de 36,99 a 44,29 $\mathrm{mm}$ e 31,82 a $35,97 \mathrm{~mm}$, com média geral de 41,89 e 34,35 $\mathrm{mm}$, respectivamente (Tabela 1).

Os frutos dos acessos A5, A6 e A7 se destacaram por apresentar os maiores comprimentos, enquanto os dos $\mathrm{A} 3 \mathrm{e}$ A8 foram os de menores comprimentos e diâmetros, que se encontram próximo aos reportados por Santos et al. (2010), com comprimento e diâmetro de 43,8 e 31,6 mm, respectivamente, porém superiores aos reportados por Carvalho et al. (2008), 38,0 e $31,0 \mathrm{~mm}$.

A massa fresca dos frutos apresentou média de $28,82 \mathrm{~g}$, com mínimo de 20,27g (A3) e máximo de 30,93g (A8), de modo que o acesso A3 diferiu significativamente $(p \leq 0,05)$ dos demais acessos. A massa do $\mathrm{A} 3$ se manteve próximo à encontrada por Carvalho et al. (2008) e Lira Júnior et al. (2005), com19,8 e 20,69 g, respectivamente. Por outro lado, a média geral, tal como a massa fresca dos demais acessos, foi bem superior à citada por Santos et al. (2010), 23,18 g em frutos do Recôncavo Sul da Bahia.

A classificação de Bosco et al. (2000) para o cajá (Spondias mombin L.) agrupa, como frutos grandes, aqueles com massa fresca superior a $15 \mathrm{~g}$, isto é, médios entre 12 e $15 \mathrm{~g}$; pequenos, os inferiores a $12 \mathrm{~g}$. Portanto, e com base nesses autores, os umbus-cajás avaliados podem ser classificados como de tamanho grande; apesar de que vale salientar, que a massa fresca média de frutos é uma característica varietal flexível dentro dos limites típicos de cada cultivar quando, os frutos 
Tabela 1. Caracterização física de frutos de acessos de umbu-cajazeira (Spondias sp.) colhidos na maturidade comercial, com coloração da casca totalmente amarela

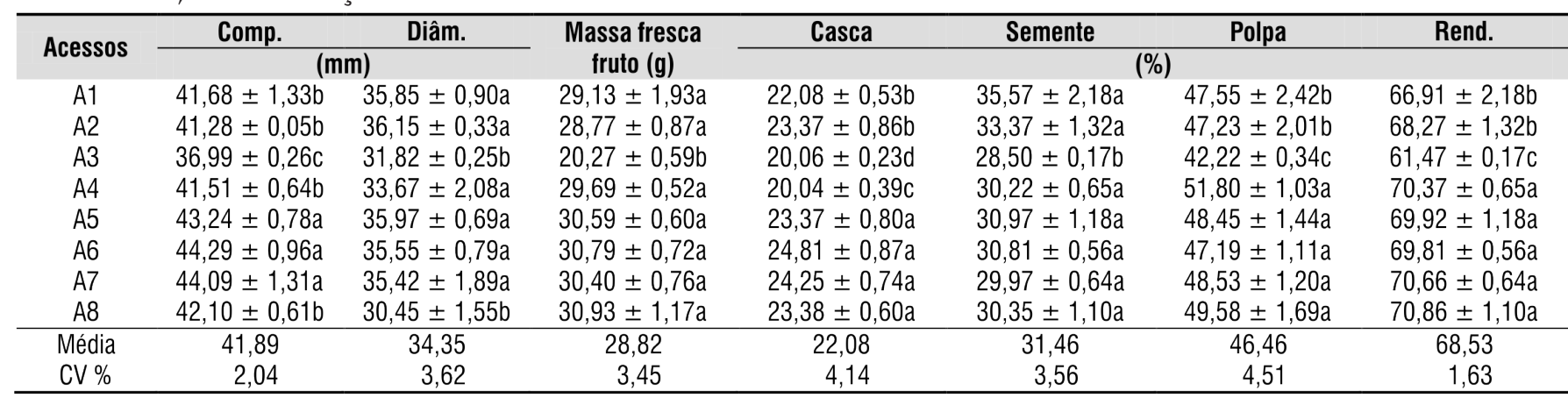

* Médias seguidas da mesma letra na coluna não diferem entre si, pelo teste de Scott-Knott a 0,05 de probabilidade

atingem o pleno desenvolvimento fisiológico, além de fatores edafoclimáticos (Chitarra \& Chitarra, 2005).

Para a percentagem de casca, os valores oscilaram entre 20,04 a $24,81 \%$, com média de $22,08 \%$. A percentagem de casca diferiu entre acessos destacando-se o A3 com menor valor em decorrência, provavelmente, do menor tamanho e da massa do fruto.

Quanto à percentagem da semente de umbu-cajá, obteve-se média de $31,46 \%$, próxima à obtida por Carvalho et al. (2008), $31,81 \%$, tendo o acesso A3 diferido $(\mathrm{p} \leq 0,05)$ dos demais acessos com a menor média, $28,50 \%$; no entanto, foi superior à reportada por Santos et al. (2010), 20,31\%. Esta característica está relacionada com a massa do fruto uma vez que os mais pesados também apresentam maior percentual de semente, evento passível de ser confirmado para o A3, que apresentou a menor massa do fruto. No processamento esta característica nos frutos é um dos principais atributos de qualidade, visto que tem influência direta no rendimento.

A percentagem de polpa está relacionada com o rendimento do fruto ambos, portanto, diretamente proporcionais. $\mathrm{O}$ rendimento médio de polpa foi igual a $68,53 \%$ destacando-se os $\mathrm{A} 5, \mathrm{~A} 6, \mathrm{~A} 7 \mathrm{e}$ A8 em virtude de apresentarem valores superiores que variaram entre 69,81 a $70,86 \%$, os quais também foram próximos aos citados por Santos et al. (2010) com $69,70 \%$ e ligeiramente inferiores aos reportados por Soares et al. (2006) $72,6 \%$, em frutos da cajazeira; mesmo assim, todos os acessos avaliados apresentaram características satisfatórias para industrialização com percentual médio de rendimento acima de $40 \%$, valor mínimo estipulado pelo Padrão de Identidade e
Qualidade (PIQ) do Ministério da Agricultura, Pecuária e do Abastecimento (MAPA) (Brasil, 1999).

O teor de sólidos solúveis (SS) dos frutos de acessos de umbu-cajazeira avaliados, diferiram significativamente entre si $(\mathrm{p} \leq 0,05)$ (Tabela 2$)$, com variação de 10,97 a 12,90\% (A3 e A2) e média de $11,86 \%$, sendo ligeiramente superiores aos de Santos et al. (2010) e Lira Júnior et al. (2005) com 10 e 10,14\%, respectivamente, embora tenham sido próximos aos citados por Lima et al. (2002) com 11,25\%.

A acidez titulável (AT), diferiu significativamente ( $p$ $\leq 0,05)$ entre os acessos tendo os A1, A3 e A4 os maiores valores com 1,15, 1,26 e 1,17\%, respectivamente (Tabela 2). Valores próximos de AT foram citados por Santos et al. (2010) e Carvalho et al. (2008) com $1,32 \%$ e 0,9 a $2,6 \%$, respectivamente. Os acessos com frutos que apresentaram AT superior a 1,00\% são os de maior interesse para a agroindústria, pois minimizam a necessidade da adição de ácido cítrico para padronização da polpa e inibir o desenvolvimento de microorganismos (Lima et al., 2002).

A relação sólidos solúveis/acidez titulável (SS/AT) variou entre 9,97 a 13,75 com média de 11,43, cujo maior valor foi obtido para o acesso A2 (Tabela 2). Em umbu-cajá maduro foi reportada variação entre 7,14 a 10,94 (Lira Júnior et al. 2005), 4,56 a 15,30 (Silva Júnior et al., 2004) e 3,80 a 7,51 (Lima et al., 2002) para frutos colhidos em diferentes estádios de maturação. Soares et al. (2006) encontraram variação de 4,9 a 16,7, com média de 10,5, em cajá. Esta relação é um parâmetro importante para avaliar a qualidade dos frutos constituindo-se numa das formas mais usuais de se avaliar o sabor por representar de modo

Tabela 2. Características físico-químicas de frutos" de acessos de umbu-cajazeira (Spondias sp.) colhidos na maturidade comercial, com coloração da casca totalmente amarela

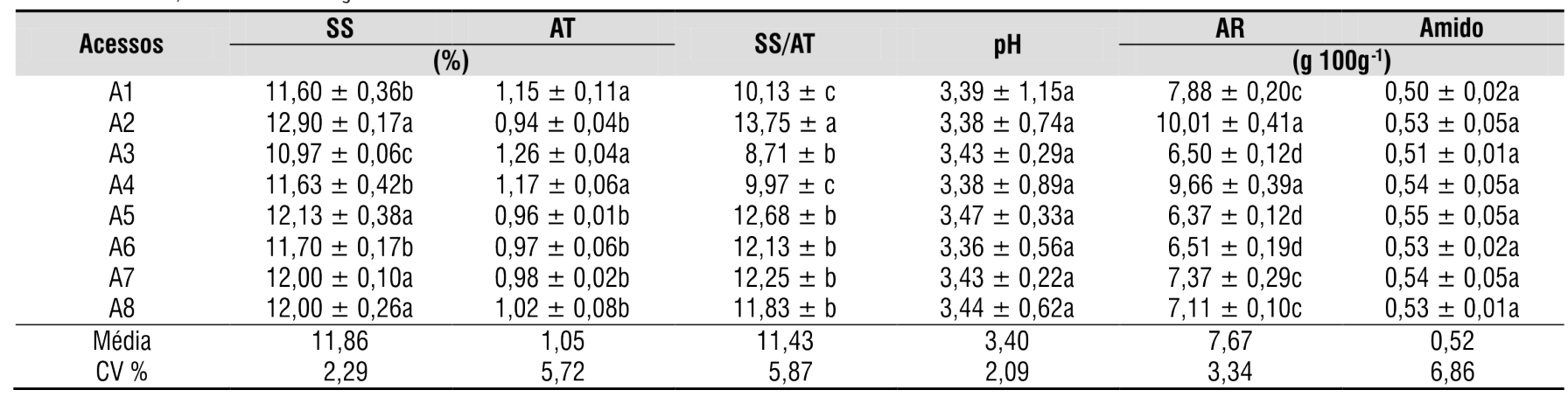

\# SS - sólidos solúveis, AT - acidez titulável, SS/AT - relação sólidos solúveis/acidez titulável

* Médias seguidas da mesma letra na coluna não diferem entre si pelo teste de Scott-Knott, a 0,05 de probabilidade 
mais significativo, o equilíbrio entre esses dois componentes devendo-se especificar o teor mínimo de sólidos solúveis e o máximo de acidez (Chitarra \& Chitarra, 2005).

$\mathrm{O}$ pH não diferiu entre frutos dos diferentes acessos, cuja média foi de 3,40 (Tabela 2). Os resultados encontrados são, entretanto, superiores aos de Santos et al. (2010) $(2,8)$, aos de Carvalho et al. (2008) $(2,40$ a 3,0) e aos de Lima et al. (2002) $(2,01$ a 2,09). No processamento de frutos o pH baixo favorece a conservação dos alimentos por dificultar o desenvolvimento de micro-organismos enquanto que, para o consumo fresco, valores de $\mathrm{pH}$ mais elevados têm maior preferência do consumidor.

Os valores de SS, pH e AT são superiores aos mínimos estabelecidos pelo Padrão de Identidade e Qualidade (PIQ) do Ministério da Agricultura Pecuária e do Abastecimento (MAPA), através da Instrução Normativa $\mathrm{n}^{\circ} .122$, de 13 de setembro de 1999, para a polpa de cajá, cujo valor mínimo é de SS 9\%, pH 2,2 e AT de $0,9 \mathrm{~g}$ de ácido cítrico $100 \mathrm{~g}^{-1}$ (Brasil,1999). O cajá foi utilizado como parâmetro de comparação por se tratar de fruto do mesmo gênero botânico e ser de mais ampla ocorrência (Soares et al., 2006), além de possuir características similares às do umbu-cajá e por este não ter legislação específica.

Os açúcares redutores do umbu-cajá diferiram ( $\mathrm{p} \leq$ $0,05)$ entre os acessos avaliados oscilando de 7,11 a 10,01

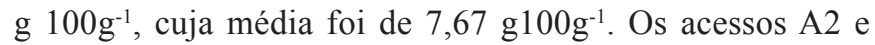
A4 apresentaram valores superiores com 10,01 e 9,66 g $100 \mathrm{~g}^{-1}$, respectivamente. Silva et al. (2011), reportaram valor

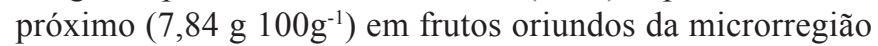
de Iguatu, CE.

Em contrapartida, Santos et al. (2010) reportaram valores

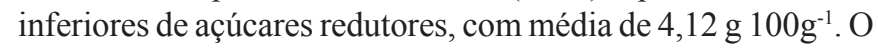
conteúdo de açúcares redutores se constitui principalmente de glicose e frutose. A quantificação do teor de açúcares individuais é importante quando se objetiva avaliar o grau de doçura do produto, haja vista, que o poder adoçante desses açúcares é variado e aumenta na sequência glicose: sacarose: frutose (Chitarra \& Chitarra, 2005).

O conteúdo de amido em frutos da umbu-cajazeira, colhidos maduros, não diferiu $(p \leq 0,05)$ entre os acessos avaliados com variação de $0,50 \mathrm{a} 0,55 \mathrm{~g}^{100 \mathrm{~g}^{-1}}$. Esses resultados corroboraram com Santos et al. (2010), que reportaram valores de $0,56 \mathrm{~g} 100 \mathrm{~g}$ ${ }^{1}$ de amido em umbu-cajá maduro proveniente do Recôncavo Sul da Bahia.

Resultados próximos também foram reportados por Silva

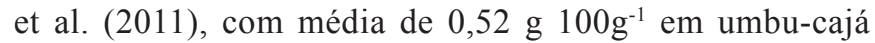
maduro. Valores inferiores ao deste trabalho, no entanto, foram encontrados por Silva (2008), em diferentes acessos de umbu-

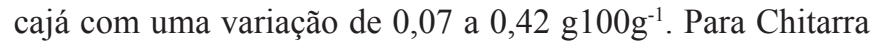
\& Chitarra (2005), o amido é o principal material de reserva energética nos vegetais em que, com a evolução da maturação dos frutos, é hidrolisado em açúcares solúveis tendo efeito perceptível no sabor e na textura.

O primeiro componente foi responsável por $60,41 \%$ da variação, o segundo por 14,4\% e o terceiro por 9,39\% (Figura 1). O CP1 permitiu separar as variáveis em dois grupos. Houve correlações positivas entre massa do fruto com o comprimento e diâmetro indicando, que as duas últimas variáveis estão diretamente relacionadas com a massa do
Tabela 3. Autovetores em três componentes principais (CP1, CP2 e CP3), das características físicas e físicoquímicasde frutos de acessos de umbu-cajazeira (Spondias sp.), colhidos na maturidade comercial

\begin{tabular}{lrrr}
\hline Comprimento & 0,330204 & $-0,092373$ & $-0,262860$ \\
Diâmetro & 0,186665 & 0,327397 & 0,037599 \\
Massa fruto & 0,337615 & $-0,019869$ & $-0,234454$ \\
\% casca & 0,342531 & $-0,088932$ & $-0,139512$ \\
\% semente & 0,284569 & 0,206173 & $-0,284404$ \\
\% polpa & 0,335521 & $-0,059278$ & $-0,217608$ \\
Rendimento (\%) & 0,323634 & $-0,100217$ & $-0,150705$ \\
SS & 0,270099 & 0,172646 & 0,522507 \\
pH & $-0,035001$ & $-0,605224$ & 0,276910 \\
AT & $-0,317839$ & 0,096564 & $-0,285837$ \\
SS/AT & 0,304549 & $-0,003195$ & 0,419083 \\
Açúcares redutores & 0,058682 & 0,571729 & 0,245214 \\
Amido & 0,234786 & $-0,295972$ & 0,192227 \\
\hline$\lambda$ & 7,85 & 1,87 & 1,22 \\
VA(\%) & 60,41 & 74,81 & 84,20 \\
\hline
\end{tabular}

$\lambda$ - Autovalor da matriz de correlaçãa; VA - Variância acumulada

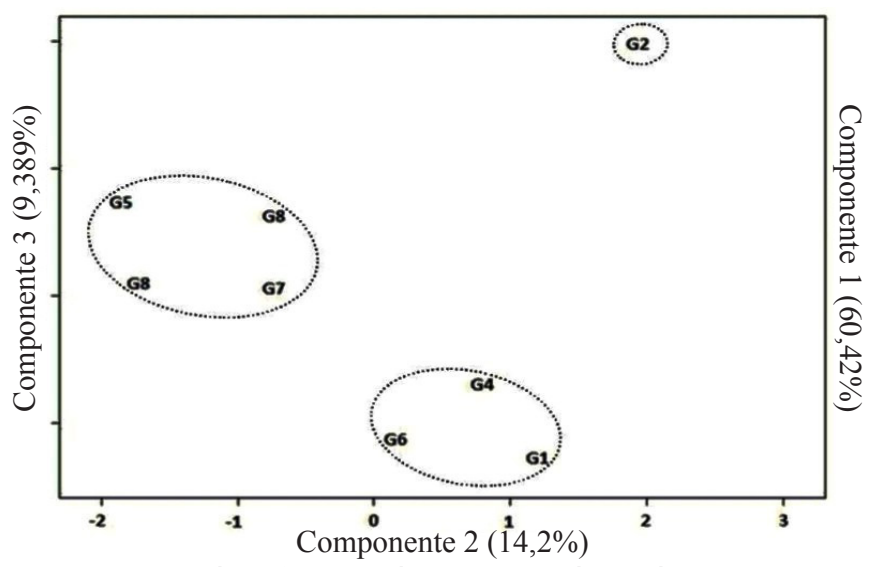

Figura 1. Classificação dos acessos de umbu-cajazeira (Spondias sp.) baseada nos escores das primeira, segunda e terceira componentes principais (CP1, CP2 e CP3)

fruto. Todavia, verifica-se que o comprimento tem influência maior na determinação da massa dos frutos de umbu-cajazeira avaliados. Correlações positivas, também foram verificadas entre percentagem de polpa e de casca e rendimento, uma vez que o rendimento é a soma dessas duas variáveis.

Os SS se correlacionaram positivamente com a relação SS/AT e amido e inversamente com a AT. A correlação entre os SS e o amido é decorrente da hidrólise do amido, que se acumula durante o desenvolvimento do fruto na planta, e inversamente com a AT, indicando que o decréscimo da acidez titulável favorece o aumento da relação SS/AT, o que também foi verificado no $\mathrm{CP} 3$.

$\mathrm{O} \mathrm{CP} 2$ está relacionado com o pH e os açúcares redutores sendo esta relação inversa, em decorrência dos ácidos orgânicos e outros compostos presentes no vacúolo contribuírem para o aumento dos açúcares durante o amadurecimento do fruto (Chitarra \& Chitarra, 2005).

A dispersão gráfica da análise de componentes principais (Figura 1), envolvendo os três componentes principais permitiu a formação de três grupos. $\mathrm{O}$ acesso 2 formou um grupo isolado destacando-se por apresentar sólidos solúveis mais elevados e maior relação SS/AT. 


\section{CONCLusÕES}

1. Com exceção do acesso A3, os demais acessos do umbu-cajazeira apresentaram características agroindustriais satisfatórias, com rendimento acima de $65 \%$ e teor de sólidos solúveis superior a 9,0, valor mínimo exigido pelo padrão de identidade e qualidade (PIQ).

2. Os frutos do acesso A2 se destacaram por apresentar sólidos solúveis e açúcares redutores mais elevados, além de relação SS/AT superior, indicando a aptidão deste acesso para consumo fresco e para processamento.

\section{Agradecimentos}

Ao CNPq (Processo 483964/2010-7) pelo auxílio financeiro e pela bolsa ao primeiro autor (Doutorado-Edital MCT/CNPq $n^{\circ}$ 27/2007, Processo n $566587 / 2008-4$ ) e à CAPES, pela bolsa (Processo: BEX 0089/11-7) do Programa de Doutorando no Brasil com Estágio no Exterior (PDEE).

\section{Literatura Citada}

AOAC - Association of Official Analytical Chemists. Official methods of analysis of international. 17.ed. Washisgton: AOAC, v.II, 2000.1018p.

Bosco, J.; Soares, K. T.; Aguiar Filho, S. P. de.; Barros, R. V. A cultura da cajazeira João Pessoa: EMEPA, 2000. 29p. Documentos, 28.

Brasil. Ministério da Agricultura, Pecuária e do Abastecimento. Instrução Normativa $\mathrm{n}^{\circ}$ 122, de 10 de setembro de 1999. Diário Oficial da República Federativa do Brasil, Brasília, 13 de setembro de 1999. Seção 1. Brasília: MAPA, 1999. p.72-76.

Brasil. Normas Analíticas do Instituto Adolfo Lutz: Métodos químicos e físicos para análise de alimentos. São Paulo: IAL, 2008. 919p.

Carvalho, P. C. L.; Ritzinger, R.; Soares Filho, W. S.; Ledo, C. A. S. Características morfológicas, físicas e químicas de frutos de populações de umbu-cajazeira no estado da Bahia. Revista Brasileira de Fruticultura, v.30, p.140-147. 2008.

Chitarra, M. I. F.; Chitarra, A. D. Pós-colheita de frutas e hortaliças: Fisiologia e manuseio. Lavras: UFLA, 2.ed., 2005. 293p.

Lima, E. D. P. A.; Lima, C. A. A.; Aldrigue, M. L.; Gondim, P. J. S. Caracterização física e química dos frutos da umbucajazeira (Spondias spp.) em cinco estádios de maturação, da polpa congelada e néctar. Revista Brasileira de Fruticultura, v.24, p.338-343. 2002.
Lira Júnior, J. S. de; Musser, R. dos S.; Melo, E.de A.; Maciel, M. I. S.; Lederman, I. E.; Santos, V. F.dos. Caracterização física e físico-química de frutos de cajá-umbu (Spondias spp.). Ciência e Tecnologia de Alimentos, v.25, p.757-761, 2005.

Ritzinger, R.; Soares Filho, W. S.; Carvalho, P. C. L. Evaluation of umbu-caja germplasm in the state of Bahia, Brazil. Crop Breeding and Applied Biotechnology, v.8, p.181-186, 2008.

Santana, I. B. B.; Oliveira, E. J. de; Soares Filho, W.S.; Ritzinger, R.; Amorim, E. P.; Costa, M. A. P. C.; Moreira, R. F. C. Variabilidade genética entre acessos de umbucajazeira mediante análise de marcadores ISSR. Revista Brasileira de Fruticultura, v.33, p.868-876, 2011.

Santos, M. B.; Cardoso, R. L.; Fonseca, A. A. O.; Conceição, M. N. Caracterização e qualidade de frutos de umbucajá (Spondias tuberosa x S. mombin) provenientes do Recôncavo Sul da Bahia. Revista Brasileira de Fruticultura, v.32, p.1089-1097. 2010.

Silva, C. R.; Koblitz, M. G. B. Partial characterization and inactivation of peroxidases and polyphenol-oxidases of umbu-cajá (Spondias spp.). Ciência e Tecnologia de Alimentos, v.30, p.790-796, 2010.

Silva, F. V. G.; Silva, S. M.; Silva, G. C.; Mendonça, R. M. N.; Alves, R. E.; Dantas. A. L. Bioactive compounds and antioxidant activity in fruits of clone and ungrafted genotypes of yellow mombin tree. Ciência e Tecnologia de Alimentos, v.32, p.685-691, 2012.

Silva, L. R.; Alves, R. E.; Aragão, F. A. S. ; Silva, S. M. ; Maia, L. K.; Nogueira, D. H. Qualidade de frutos de genótipos de umbu-cajazeiras (Spondias sp.) oriundos da Microrregião de Iguatu, CE. Scientia Plena, v.7, p.1-7, 2011.

Silva Júnior, J. F.; Bezerra, J. E. F.; Lederman, I. E.; Alves, M. A.; Melo Neto, M. L. Collecting, ex situ conservation and characterization of "cajá-umbu" (Spondias mombim $\mathrm{x}$ Spondias tuberosa) germ-plasm in Pernambuco State, Brazil. Genetic Resources and Crop Evolution, v.51, p.343349, 2004.

Soares, E. B.; Gomes, R. L. F.; Carneiro, J. G. de M.; Nascimento, F. N. do; Silva, I. C. V.; Costa, J. C. L. da. Caracterização física e química de frutos de cajazeira. Revista Brasileira de Fruticultura, v.28, p.518-519, 2006.

Souza, F. X.; Costa, J. T. A.; Lima, R. N.; Crisóstomo, J. R. Crescimento e desenvolvimento de clones de cajazeira cultivados na Chapada do Apodi, Ceárá. Revista Brasileira de Fruticultura, v.28, p.414-420, 2006. 